\title{
Investigation of a sunspot complex by time-distance helioseismology
}

\author{
A. G. Kosovichev ${ }^{1}$ and T. L. Duvall $\mathbf{J r}^{2}$ \\ ${ }^{1}$ Stanford University, Stanford, CA 94305, USA \\ ${ }^{2}$ Solar Physics Laboratory, Goddard Space Fight Center, NASA, Greenbelt, MD 20771, USA
}

\begin{abstract}
Sunspot regions often form complexes of activity that may live for several solar rotations, and represent a major component of the Sun's magnetic activity. It had been suggested that the close appearance of active regions in space and time might be related to common subsurface roots, or "nests" of activity. EUV images show that the active regions are magnetically connected in the corona, but subsurface connections have not been established. We investigate the subsurface structure and dynamics of a large complex of activity, NOAA 10987-10989, observed during the SOHO/MDI Dynamics run in March-April 2008, which was a part of the Whole Heliospheric Interval (WHI) campaign. The active regions in this complex appeared in a narrow latitudinal range, probably representing a subsurface toroidal flux tube. We use the MDI full-disk Dopplergrams to measure perturbations of travel times of acoustic waves traveling to various depths by using time-distance helioseismology, and obtain sound-speed and flow maps by inversion of the travel times. The subsurface flow maps show an interesting dynamics of decaying active regions with persistent shearing flows, which may be important for driving the flaring and CME activity, observed during the WHI campaign. Our analyses, including the seismic sound-speed inversion results and the distribution of deep-focus travel-time anomalies, gave indications of diverging roots of the magnetic structures, as could be expected from $\Omega$-loop structures. However, no clear connection in the depth range of $0-48 \mathrm{Mm}$ among the three active regions in this complex of activity was detected.
\end{abstract}

Keywords. Sun: helioseismology, sunspots, Sun: interior, Sun: magnetic field

\section{Introduction}

Local helioseismology provides important insight into the subsurface structure and dynamics of emerging magnetic flux, formation, evolution and decay of sunspots and active regions. Methods of local helioseismology and acoustic tomography, based on measurements and inversion of acoustic travel times, are intensively developed and tested via numerical simulations (for a recent review see, Kosovichev 2010). These methods provide important insights in the subsurface structure and dynamics of sunspots, which are important for understanding the origins of solar magnetism. In particular, our previous results obtained by a time-distance helioseismology techniques (Duvall et al. 1993) revealed significant changes in the subsurface flow patterns during the life cycle of active regions: strong diverging flows during the flux emergence, formation of localized converging flows around stable sunspots, and dominant outflows during the decay (Kosovichev \& Duvall 2006; Kosovichev 2009). The sound-speed images indicate that the magnetic flux gets concentrated in strong field structures just below the surface during the sunspot formation, but the seismic perturbations of large sunspots extend at least up to 20-30 $\mathrm{Mm}$, indicating that sunspots have deep roots.

A complex of decaying sunspots, NOAA 10987-10989, was observed by SOHO/MDI in March 2008 during Whole Heliospheric Interval (WHI) campaign. Three active regions 


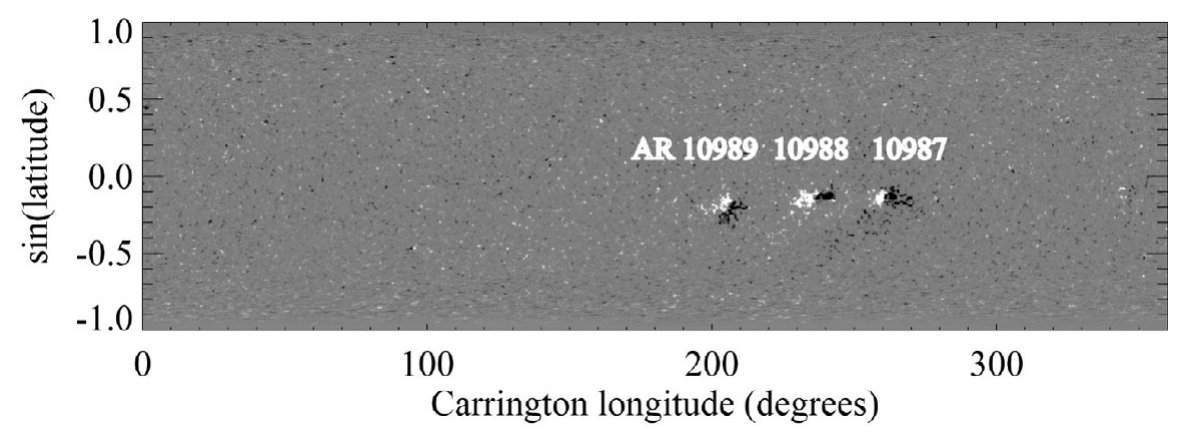

Figure 1. Synoptic magnetic field map from SOHO/MDI for Carrington rotation 2068. The grayscale shows the line-of-sight magnetic field saturated at $\pm 100 \mathrm{G}$.

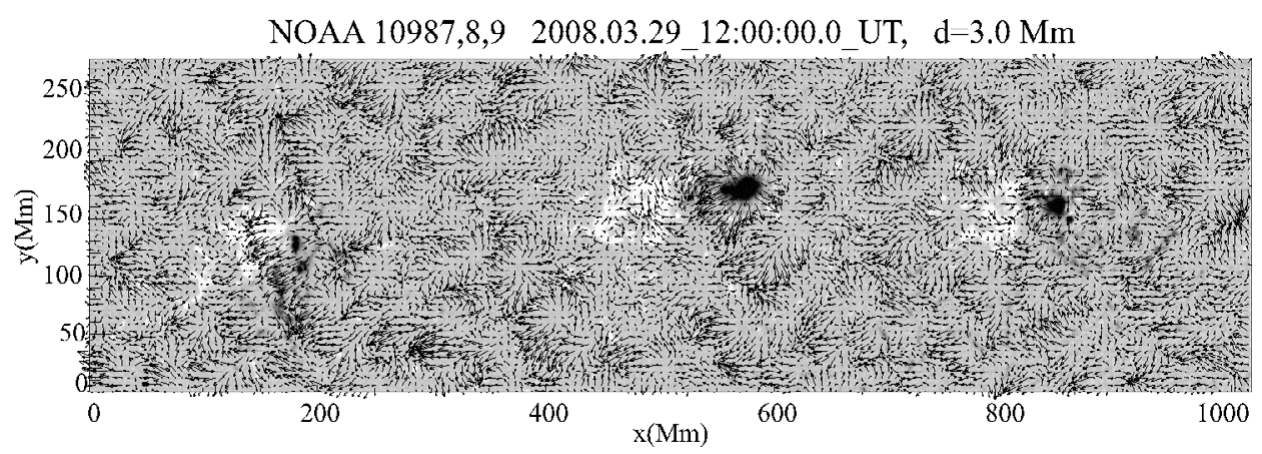

Figure 2. A map of subsurface flows at depth $3 \mathrm{Mm}$ (arrows) over the corresponding photospheric magnetogram for all three active regions, observed on March 29, 2008, 12:00 UT. The longest arrows correspond to $0.5 \mathrm{~km} / \mathrm{s}$. The magnetic field scale is from -100 to $500 \mathrm{G}$.

appeared in a narrow latitudinal and longitudinal range (Fig. 1). This suggests that they probably originated from a common subsurface nest of activity.

\section{Data Analysis and Results}

For the analysis we used full-disk Dopplergrams from SOHO/MDI (Scherrer et al. 1995) obtained with 1-min cadence and $2 \mathrm{arcsec} /$ pixel resolution. The time-distance data analysis and inversion procedure is described by Duvall et al. (1997); Kosovichev (1996); Kosovichev \& Duvall (1997). The acoustic travel-time maps were calculated for 12 distance ranges (annuli), from 0.78 to 11.76 heliographic degrees (or from 9.48 to 142.85 Mm) for fifteen 8-hour intervals during the period of March 27 - April 1, 2008. We used both the surface-focus and deep-focus measurement schemes (Duvall 1998). This coverage allowed us to investigate evolution of this decaying complex of activity.

Figure 2 shows a subsurface flow map of all three active regions at the depth of $3 \mathrm{Mm}$. The flow pattern shows supergranulation flows and strong diverging flows around sunspots of these active regions. The diverging flows are observed in the whole range of depth, $0-12 \mathrm{Mm}$ obtained in our inversions. At greater depths the inversion results become too noisy. We did not attempt to recover the flow patterns at greater depths in this work. The dominance of diverging flows for the decaying sunspots is consistent with our previous results (Kosovichev \& Duvall 2006; Kosovichev 2009).

The structures of the subsurface flows in AR 10989 and 10988 are shown in more detail in Figure 3. An interesting feature is strong shearing flows associated with the diverging 

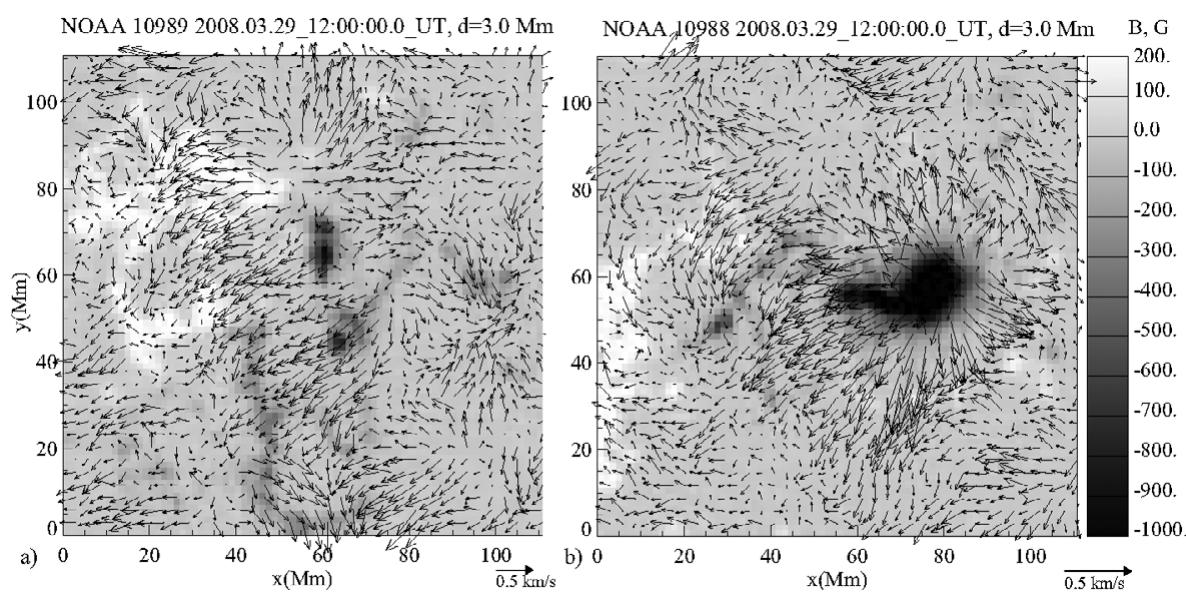

Figure 3. Subsurface flow maps at depth $3 \mathrm{Mm}$ in active regions a) 10989, and b) 10988. The background images are the corresponding photospheric magnetograms.
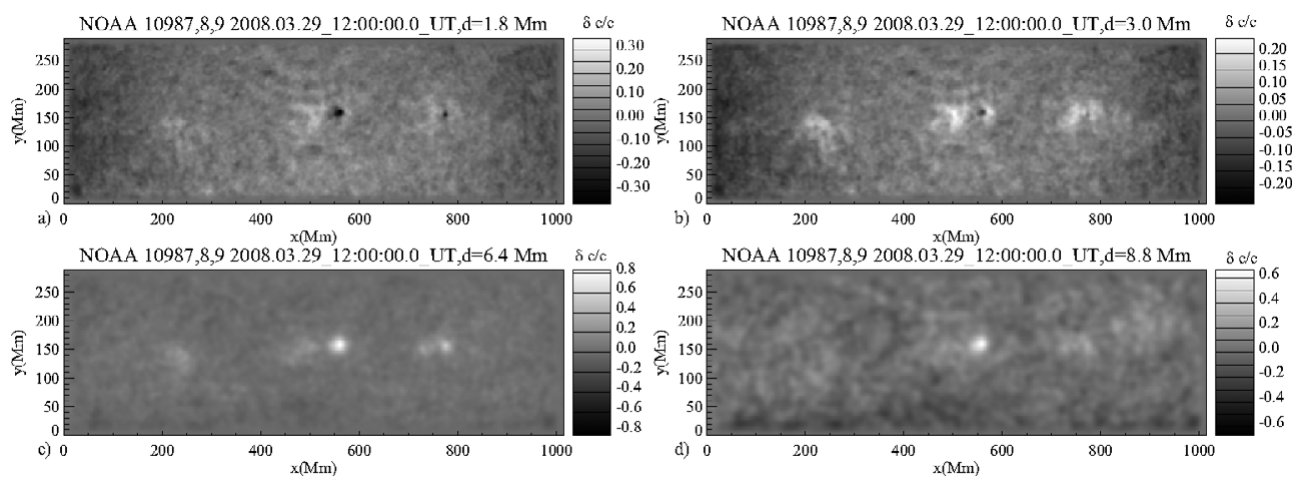

Figure 4. Maps of subsurface sound-speed variations beneath the active regions, NOAA 10987 - 9, at depth: a) $1.8 \mathrm{Mm}$, b) $3 \mathrm{Mm}$, c) $6.4 \mathrm{Mm}$, and d) $8.8 \mathrm{Mm}$.

flows of the decaying sunspots. These flows may be important for understanding the flaring and CME activity of decaying regions. In this case, during the WHI campaign several strong CMEs were detected from AR 10989, which was decaying most rapidly.

Our inspection of the flow maps did not reveal any large-scale pattern, which can be common for these active regions and indicate on their subsurface connections. Therefore, we investigated also helioseismic sound-speed maps, obtained by inversion of the surface-focus travel-time variations, using the ray-path approximation (Kosovichev 1999). Samples of the seismic sound-speed maps are shown in Fig. 4. Interestingly enough, a shallow layer of negative sound-speed variation, previously reported for isolated sunspots (e.g. Couvidat et al. 2006a,b; Kosovichev et al. 2000; Zhao et al. 2010), is observed only for the leading sunspots of AR 10988 and 10987. The seismic structure of the sunspot in AR 10989 showed only positive variations, probably because it was mostly decayed.

These maps, in which the seismic structure is clearly seen up to $12 \mathrm{Mm}$ depth, also did not reveal potential subsurface links among the active regions. Therefore, we studied averaged properties in order to obtain a better signal-to-noise ratio at greater depths. Figure $5 a$ shows the depth structure of the sound-speed inversions averaged over a narrow range of latitude. The seismic structures can be traced up to the depth of $24 \mathrm{Mm}$. It 

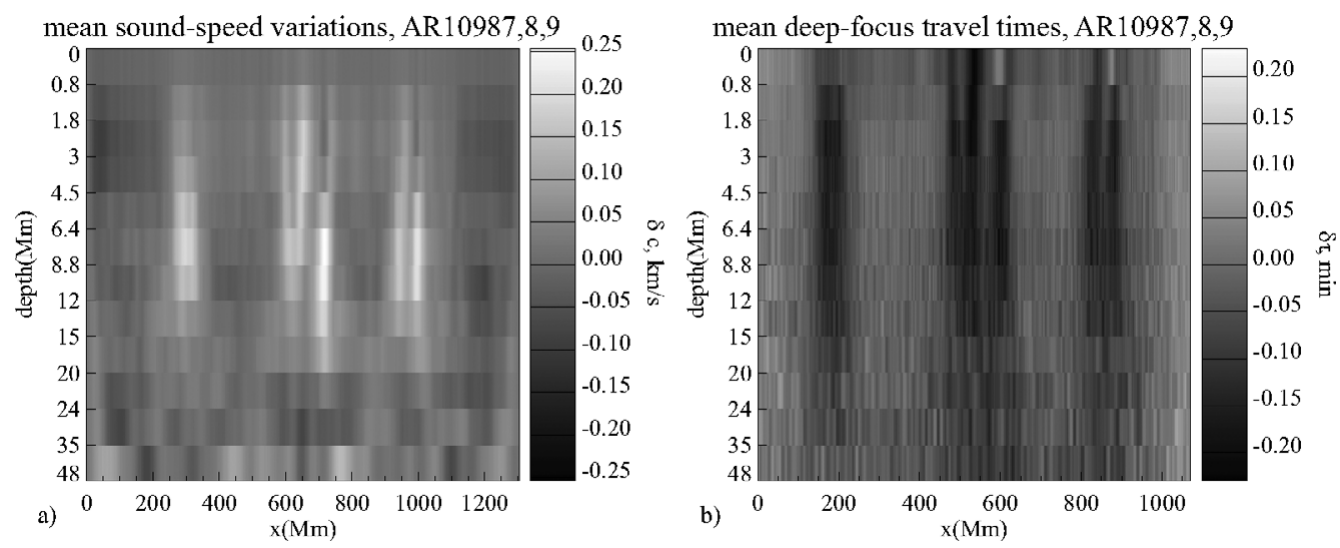

Figure 5. a) Sound-speed variations, averaged over a narrow range of latitude including all three active regions, as a function of longitude ( $x$-coordinate) and depth. b) Travel-time variations calculated by using the deep-focus scheme and averaged over a narrow range of latitude, as a function of longitude and the focus depth.

seems that the tube-like structures become more inclined and diverge from each other with depth, like this can be expected from $\Omega$-loop structures, but this evidence is weak. From these inversion data we cannot make a conclusion about the subsurface connections.

In addition, we attempted to detect the links directly from the deep-focus travel time anomalies. Figure $5 b$ shows the deep-focus travel time perturbations (relatively to the quiet Sun) averaged over a range of latitudes including the active regions, as a function of longitude and the focus-point depth. The depth distribution of these travel-times is qualitatively similar to the sound-speed inversion results from the different (surface-focus) type of measurements. The deep-focus data allows us to trace the subsurface structures in deeper layers, up to $48 \mathrm{Mm}$. They also indicate divergence of the active region structures, which may be an evidence that these are parts of a $\Omega$-loop toroidal structure. But this remains uncertain and requires further investigation.

\section{Conclusions}

We have used data from SOHO/MDI to investigate seismic sound-speed structures and mass flows in the solar interior, associated with a complex of three active regions. We found that all sunspots in this complex were decaying and were surrounded by strong deep outflows and shearing flows, which could be important for initiation of CMEs observed during this period. Our time-distance helioseismology analyses, including seismic soundspeed inversions of the surface-focus travel-time measurements and deep-focus travel-time anomalies, provided indications of diverging roots of the magnetic structures as this could be expected from $\Omega$-loop structures, but were unable to detect connections in the depth range of $0-48 \mathrm{Mm}$ among the three active regions in this complex.

\section{References}

Couvidat, S., Birch, A. C., \& Kosovichev, A. G. 2006a, Astrophys. J., 640, 516

Couvidat, S., Birch, A. C., Rajaguru, S. P., \& Kosovichev, A. G. 2006b, in IAU Symposium, Vol. 233, Solar Activity and its Magnetic Origin, ed. V. Bothmer \& A. A. Hady, 75-76

Duvall, Jr., T. L. 1998, in ESA Special Publication, Vol. 418, Structure and Dynamics of the Interior of the Sun and Sun-like Stars, ed. S. Korzennik, 581

Duvall, Jr., T. L., Jefferies, S. M., Harvey, J. W., \& Pomerantz, M. A. 1993, Nature, 362, 430 
Duvall, Jr., T. L., Kosovichev, A. G., Scherrer, P. H., et al. 1997, Solar Phys., 170, 63

Kosovichev, A. 2010, Solar Phys., submitted

Kosovichev, A. G. 1996, Astrophys. J. Lett., 461, L55

-. 1999, Journal of Computational and Applied Mathematics, 109, 1

-. 2009, Space Science Reviews, 144, 175

Kosovichev, A. G. \& Duvall, T. L. 2006, Space Science Reviews, 124, 1

Kosovichev, A. G., Duvall, Jr., T. L. ., \& Scherrer, P. H. 2000, Solar Phys., 192, 159

Kosovichev, A. G. \& Duvall, Jr., T. L. 1997, in Astrophys. Space Sci. Lib., v.225, SCORe'96 :

Solar Convection and Oscillations and their Relationship, 241-260

Scherrer, P. H., Bogart, R. S., Bush, R. I., et al. 1995, Solar Phys., 162, 129

Zhao, J., Kosovichev, A. G., \& Sekii, T. 2010, Astrophys. J., 708, 304 